\title{
Vers une abolition de la vivisection.
}

\author{
Laurence Harang ${ }^{1}$
}

\section{Sommaire}

\section{Introduction}

I. L'expérimentation est nécessaire à la connaissance biologique

1. Une brève histoire de la physiologie

2. Les limites de la physiologie

II. L'utilitarisme et la science : la liberté du chercheur doit-elle être absolue ?

1. La science est au service de la société

2. L'utilité contre la cruauté : le combat des antivivisectionnistes

\section{La protection des animaux d'expérimentation}

1. Le droit européen et les mesures relatives à la protection des animaux

2. Protection ou exploitation des animaux de laboratoire?

\section{Vers une abolition de la vivisection}

1. L'animal n'est pas le modèle biologique de l'homme

2. Des droits pour les animaux?

\section{Conclusion}

\section{Introduction}

La vivisection ou l'expérimentation animale a toujours été l'objet de polémique de I'Antiquité grecque en passant par le XIXe siècle avec les expérimentateurs comme Bernard, Flourens, Magendie jusqu'aux laboratoires privés et publics du XXle siècle! Selon une définition courante, la vivisection est une intervention chirurgicale sur un animal conscient à des fins de connaissance. Evidemment, il faut distinguer deux formes d'expérimentation; la première a le souci de guérir tandis que la seconde vise

${ }^{1}$ Docteur et Professeur de Philosophie à Toulon (France) 
à augmenter le savoir. Il est vrai que pour des raisons éthiques, la recherche sur les animaux est obligatoire depuis Nuremberg puisque, au nom du consentement éclairé, le citoyen doit être informé et libre de ses décisions.

Il est évident que l'on ne peut admettre l'acceptation " neutre " du terme de vivisection tant par l'idéologie véhiculée que par la cruauté de sa pratique. L'expérimentation animale heurte en effet notre sensibilité morale par les souffrances infligées aux êtres sensibles. On pourrait se demander en conséquence pourquoi cet art médical - tel qu'il est entendu par les vivisecteurs au XIXe siècle - connaît une telle apogée au temps de Claude Bernard. II faut donc constater que la médecine expérimentale a besoin pour son prestige d'être reconnue socialement. La vivisection, à ce titre, constitue la norme de la physiologie au XIXe siècle : c'est ainsi que les expérimentateurs, à l'Académie des sciences, livrent au public, le secret du fonctionnement du corps. Mais il faut, pour expliquer l'apogée de la science expérimentale, s'interroger sur l'idéologie scientiste qui domine la recherche durant le XIXe siècle.

Au nom du Progrès, de la Science, de la Raison, la méthode expérimentale acquiert ses titres de noblesse : par sa maîtrise du corps de l'animal, le vivisecteur expose son art et son savoir. C'est ainsi que la connaissance des lois de la matière permettra de se rendre " comme maître et possesseur de la nature " selon le fameux adage cartésien. L'idéal républicain s'incarne dans le rationalisme expérimental, dans la conquête du savoir. II est de fait évident que le pouvoir sur les animaux viviséqués (les vertébrés) n'est autre qu'un pouvoir sur les êtres vulnérables. Car la science en tant qu'elle impose de nouvelles valeurs entend faire fi des sentiments, de la compassion. L'idéal républicain, pourtant hérité des Lumières, abandonne toute aspiration à la justice, à l'égalité. Or, selon les antivivisectionnistes, le souci pour la justice sociale concerne aussi bien les catégories opprimées que les êtres sensibles comme les animaux.

Le combat contre la vivisection est dès lors au XIXe siècle une lutte politique, une lutte pour la justice. C'est ainsi que les antivivisectionnistes se réclament du mouvement progressiste et entendent bien renouer avec les idéaux des Lumières. Parmi eux se trouvent des féministes, des socialistes comme Marie Huot, Anna Kingsford et des écrivains célèbres comme Victor Hugo. II faut donc bien reconnaître que la souffrance animale est équivalente à la douleur humaine : $c^{\prime}$ est en luttant contre l'esclavage que les hommes parviendront à une égalité de droits; c'est en dénonçant les abus de la vivisection que les êtres vulnérables seront pris en considération. L'enjeu de la lutte contre la vivisection est de mettre fin à une conception positiviste et matérialiste de la science au nom d'une conception spirituelle de la vie. Ces conceptions philosophiques partagées par certaines féministes adeptes d'un mouvement de théosophie reposent sur l'idée d'une Unité des êtres vivants : il n'y a pas en ce sens une supériorité de I'homme sur l'animal. Cette critique d'un humanisme philosophique se retrouve notamment au XXe siècle par le rejet de toute forme de "spécisme »: Peter Singer, 
auteur de "La libération animale ${ }^{2}$, refuse toute " discrimination " entre les espèces. " Il apparaît donc que les mesures de protection législatives à propos des animaux d'expérimentation au XXe-XXle siècle ne peuvent abroger la souffrance des êtres sensibles. C'est pourquoi il semble, en raison des progrès de la science, que l'abolition de la vivisection constitue une évidence !

Présentons le plan de notre article : dans une première partie, nous montrerons en quoi la vivisection est un art nécessaire à la constitution de la médecine expérimentale selon Claude Bernard. Dès lors, la physiologie devient la reine des sciences biologiques: l'expérimentation sur les animaux permet selon les vivisecteurs de parvenir à une connaissance du fonctionnement du corps. Toutefois, la connaissance de la vie ne se réduit pas nécessairement à l'anatomie ; le vivant possède une propre spécificité. Il est donc important de comprendre en quoi le matérialisme de $\mathrm{Cl}$ Bernard s'oppose à une conception vitaliste du vivant.

Ensuite, dans une seconde partie, nous nous interrogerons sur le succès de la vivisection au XIXe siècle : Jean-Yves Bory ${ }^{3}$ montre avec beaucoup de justesse que la vivisection incarne l'idéal républicain par excellence : " le rationalisme expérimental " participe du Progrès, de la conquête de la matière par le médecin. II faut toutefois souligner que les idéaux républicains issus de la Révolution française ont pour conséquence l'exploitation tant humaine qu'animale. En effet, au nom du Progrès, la misère du prolétaire semble être une conséquence inéluctable du développement des forces économiques; quant à l'animal, il est tout au plus un instrument à utiliser ; et c'est pourquoi CL Bernard ne manifeste aucun scrupule à faire un usage scientifique d'un être sensible. II semble donc que la vivisection puisse contribuer au prestige de la médecine expérimentale, à sa reconnaissance sociale. Mais il est nécessaire de distinguer le prestige que confère une activité de son utilité. Or, dans le domaine de la santé, la vivisection pose un grave problème moral; elle ne fait qu'augmenter les souffrances des animaux à seule fin de satisfaire l'amour-propre des chercheurs. C'est pourquoi, la lutte contre la vivisection est une lutte politique, pour la justice sociale. Le mouvement antivivisectionniste, comme nous l'affirmions, au début de cette introduction, combat l'injustice, l'inégalité en vertu de la reconnaissance de la valeur des êtres vulnérables. En conséquence, le combat pour les animaux est un combat pour les valeurs morales telles que la compassion et l'empathie. Car il est injustifié moralement de faire de la vivisection un mal nécessaire.

Enfin dans une troisième partie, nous nous demanderons si la protection des animaux d'expérimentation est garantie depuis le XXe - XXle siècle. II est vrai qu'il existe une législation en Europe et en France. Mais à notre sens, il est incohérent de défendre à la fois le bien-être des animaux et le maintien de la vivisection. Car le sacrifice d'animaux

\footnotetext{
${ }^{2}$ La libération animale, Trad L Rousselle, Grasset 1993.

${ }^{3}$ Science et patience; la polémique sur la vivisection au XIXe siècle ;EHESS 2010.
} 
la souffrance des vertébrés ne fait aucun doute - ne peut s'autoriser d'aucun compromis. De la même manière, il serait absurde de faire des droits de l'homme des variables d'ajustement à l'aune des circonstances; car, le droit à la vie est un droit inaliénable, sacré. C'est pourquoi, l'absence de statut juridique de l'animal est à l'origine de son exploitation.

Dans une dernière partie, nous montrerons que l'abolition de la vivisection est possible et souhaitable. La science du XXIe - ainsi que le soutient le comité scientifique "Antidote » - est en mesure de proposer des méthodes substitutives : un programme de toxicologie a été testé à partir de cellules humaines; ensuite, la modélisation informatique permet de se représenter les différentes parties du corps humain.

Il est essentiel, au nom du respect et de la dignité animale de mettre fin à une barbarie d'un autre temps, la vivisection.

\section{I. l'expérimentation est nécessaire à la connaissance biologique}

1. Une brève histoire de la physiologie

De Claude Bernard, médecin et spécialiste de physiologie (1813-1878), on connaît les travaux dans le mécanisme de la sécrétion interne du glucose dans le foie, l'action du curare dans l'action de paralysie des membres d'une grenouille...Bref, $\mathrm{Cl}$ Bernard a fait de la physiologie la science de la biologie expérimentale. C'est pourquoi, il ne peut y avoir une science digne de ce nom sans expérimentation. C'est ainsi qu'il faut insister sur la démarche expérimentale de la médecine : le recherche en biologie ne doit servir que la médecine, autrement ce serait un art superflu; l'expérimentation permet de découvrir des fonctions biologiques.

La vivisection animale pour ses partisans au XIXe siècle représente " l'intervention chirurgicale, à des fins de connaissance, sur un animal conscient. " Le mot "vivisection » provient en latin du terme "vivus » qui désigne l'action de couper, sectionner les parties d'un corps vivant sans anesthésie. Aujourd'hui, la vivisection désigne les expériences invasives pratiquées sur des animaux vivants. II reste que les méthodes utilisées, en dépit de l'exigence d'assurer le bien-être de l'animal, sont cruelles.

CL Bernard découvre par l'action d'un poison, le curare, la séparation anatomique du système nerveux: sur des animaux vivants expérimentés, le médecin prouve que la section des nerfs rachidiens antérieurs produit la paralysie du mouvement alors que ce n'est pas la même chose pour les nerfs rachidiens supérieurs. En se livrant à de telles expériences, Bernard comme Magendie peuvent ainsi tester la sensibilité, le mouvement des animaux en leur coupant les racines rachidiennes antérieures et 
postérieures. II est curieux de constater l'idée selon laquelle la connaissance du vivant reposerait sur la découverte de son anatomie et de son organisation biologique; autant dire que $\mathrm{CL}$ Bernard partage la croyance selon laquelle les phénomènes de la vie résultent d'un déterminisme physico-chimique : chaque être vivant serait ainsi organisé d'une manière spécifique. C'est pourquoi, la vivisection donne à la physiologie ses lettres de noblesse. La médecine expérimentale, telle qu'elle est inaugurée par CL Bernard, participe à la connaissance du milieu intérieur de chaque espèce. C'est donc au cœur des laboratoires que le médecin prétend parvenir à une meilleure compréhension du vivant sur " le terrain fétide et palpitant de la vie. »

Tentons de comprendre sur quoi repose l'intuition de ce médecin au XIXe siècle :

" On n’a pu concevoir les lois de la matière brute qu'en pénétrant dans les corps ou dans les machines inertes, de même on ne pourra arriver à connaître les lois et les propriétés de la matière vivante qu'en disloquant les organismes vivants pour s'introduire dans leur milieu intérieur. Il faut donc nécessairement avoir disséqué sur le mort, disséquer sur le vif, pour mettre à découvert et voir fonctionner les parties intérieures ou cachées de l'organisme; c'est à ces sortes d'opérations qu'on donne le nom de vivisection, et sans ce mode d'investigation, il n'y a pas de physiologie ni de médecine expérimentale possible ${ }^{4} . »$

La vivisection semble être au service d'une connaissance de la vie. En tant que tel, il n'y a aucun respect du corps puisqu'il est à comprendre comme le support de phénomènes réglés et déterminés. En un sens, la perspective de $C L$ Bernard est à la fois matérialiste et physiologique : la compréhension des lois de la matière permettra de voir " les parties intérieures ou cachées de l'organisme. " De là se manifeste le désir d'une science puissante au service des intérêts de l'humanité ; la question est bien-sûr de savoir si de telles expérimentations peuvent contribuer au progrès de la médecine comme le pense le vivisecteur. Pourtant $C L$ Bernard est persuadé que les sciences physico-chimiques permettent " la conquête de la nature brute " comme il le souligne dans les Leçons sur les phénomènes de la vie communs aux animaux et aux hommes: il faut donc d'une certaine manière revendiquer une certaine forme de positivisme (fonder la science sur des faits expérimentaux) et accepter le mécanisme cartésien. Car le propre de cette "science physiologique " est d'agir sur les êtres vivants et de comprendre ainsi leur fonctionnement comme les physiciens maîtrisent les phénomènes naturels.

Il est vrai que l'on ne peut réduire la science physiologique de CL Bernard à un simple mécanisme selon l'intuition cartésienne. Toutefois, des analogies subsistent entre les deux méthodes, d'autant plus que le vivisecteur reconnaît l'héritage cartésien. Ainsi,

\footnotetext{
${ }^{4}$ Introduction à l'étude de la médecine expérimentale ; GF, 1966.
} 
dans la célèbre lettre au Marquis de Newcastle, le philosophe défend l'idée que l'animal est une sorte d'automate :

" Je sais bien que les bêtes font beaucoup de choses mieux que nous, mais je ne m'en étonne pas ; car cela sert à prouver qu'elles agissent naturellement et par ressort, ainsi qu'une horloge, laquelle montre bien mieux l'heure qu'il est que notre jugement ne nous l'enseigne ${ }^{5}$. "

L'animal en effet est dépourvu de pensées et de langage, car les mots proférés ne témoignent d'aucune intelligence. L'animal donc par son incapacité à exprimer une pensée est au tout plus régi par des automatismes, merveilleuses créatures divines ! Sans doute, Descartes reconnaît dans sa correspondance l'idée d'un mystère de l'animal. Mais le fait de priver la bête d'émotions et de pensées a pour conséquence de faire de lui un automate incapable de ressentir ni douleur ni plaisir. II est évident que le fait de retirer pensée et intelligence à l'animal en fait de lui un objet pour la science $^{6}$. Et les horreurs de la vivisection sur l'animal au XIXe siècle témoigneront de l'absence de sensibilité à la douleur en vertu d'une idéologie du Progrès de la science comme nous aurons l'occasion d'y revenir. Il est vrai que la différence d'essence entre I'homme et l'animal autorise - pour reprendre une expression d'Elisabeth de Fontenay - une "escalade expérimentale.» Haller au XVIIle siècle démontre sa théorie de I'irritabilité par la pratique de la vivisection afin de " contribuer à l'utilité du genre humain ${ }^{7} . "$

Au nom des impératifs de la Science, au nom du Progrès (lesquels constituent des idéologies), CL Bernard, Magendie, Flourens s'octroient le droit de pratiquer des expérimentations sans limite pour le bien de l'humanité. C'est ainsi que CL Bernard défend ses « convictions » :

" Le physiologiste n'est pas un homme du monde, c'est un savant, c'est un homme qui est saisi et absorbé par une idée scientifique qu'il poursuit ${ }^{8}$." Peu importe les souffrances, les cris, les mutilations subies par les animaux, poursuit l'auteur dans la suite de son propos. L'expérimentateur, sûr de son bon droit, refuse de polémiquer avec les opposants de son époque. Pour lui, il s'agit de délivrer l'humanité des préjugés de son temps au nom du bien-fondé de la vivisection. Car nier l'expérimentation sur l'animal consisterait à nier la médecine expérimentale, c'est-à-dire nier la médecine scientifique. L'argument défendu par CL Bernard est de justifier l'exploitation des animaux de laboratoire par le fait que les bêtes dans tous les usages de la vie sont au

\footnotetext{
${ }^{5}$ Lettre au Marquis de Newcastle, La Pléiade, Euvres et Lettres ; 1953.

${ }^{6}$ Notons qu'Harvey au XVIIe siècle déduit la circulation du sang par la vivisection des mouvements du cœur ; il n'hésite pas non plus à ouvrir le ventre de biches vivantes afin de valider sa théorie sur le foetus !

7 « La bête est sans raison », Elisabeth de Fontenay dans $\underline{\text { Critique, }} 1978$.

${ }^{8}$ Op.cité note 1.
} 
service de l'homme. Mais c'est sans aucun doute un argument fallacieux que de justifier l'exploitation par l'exploitation. On pourrait de ce fait accepter l'idée de l'expérimentation des condamnés à mort pour le bien-être de l'humanité ${ }^{9}$ ! II reste que $\mathrm{CL}$ Bernard poursuit ses recherches sur le sucre dans les tissus organiques aussi bien chez les vipères, les singes que chez les hommes guillotinés!

Toutefois, on peut se demander si la connaissance de la vie est susceptible d'être réduite à la compréhension des fonctions organiques d'un animal en particulier. N'estce pas oublier que la spécificité du vivant ne se laisse enfermer ni dans des mécanismes physico-chimiques ni dans la découverte des fonctions des organes ?

\section{Les limites de la physiologie}

II est vrai que la vivisection au XIXe siècle a pour conséquence la suprématie de la physiologie; elle devient ainsi la norme des sciences biologiques. La plupart des vivisecteurs sont par définition matérialistes et positivistes : c'est par les lois de la matière qu'une compréhension de la vie est possible. II est curieux d'ailleurs de remarquer que l'attention portée à la sensibilité du vivant (sensibilité des tissus) laisse le chercheur indifférent à la douleur de l'animal ! Il reste que la bête est précisément considérée comme un automate dont il s'agirait de découvrir les mécanismes secrets.

Si la physiologie de CL Bernard entend conquérir son autonomie à l'égard des sciences physico-chimiques, il n'en reste pas moins que " la vie c'est la mort " autrement dit un processus de dégradation de la matière. Or précisément, le vitalisme doctrine opposée au matérialisme semble contester le caractère réducteur du vivant aux lois physicochimiques. La vie est en effet caractérisée par une force vitale. Sans entrer dans le débat entre les partisans du mécanisme et du vitalisme, on peut remarquer que le vitalisme d'un Bichat (1771-1801) s'illustre par la formule célèbre: la vie est "l'ensemble des fonctions qui s'opposent à la mort "; autrement dit, la vie n'est pas simplement une dégradation de la matière. Si le vivant est animé d'une force vitale, cela fait de lui un sujet individuel et spécifique. Or, comment est-il possible d'établir des analogies entre des individus d'une même espèce voire $d^{\prime}$ 'une autre espèce comme I'homme ? C'est ainsi que l'on peut établir les limites de la physiologie et par voie de conséquence de la vivisection.

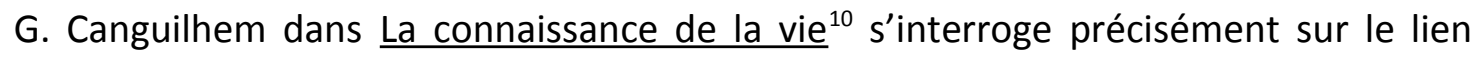
entre la méthode expérimentale et la physiologie. Ainsi, lors de fractures osseuses, il n'existe pas de stade cartilagineux chez l'homme contrairement à l'animal. Le philosophe des sciences est en mesure de défendre trois principes qui ont pour but de

\footnotetext{
9 Ajoutons que de telles pratiques existaient jusqu'au XIXe siècle puis ont été abandonnées par souci de moralité.

10" Vrin, 1980.
} 
promouvoir la spécificité du vivant. D'abord, en vertu de l'individualisation du vivant, il n'existe aucune comparaison dans l'identité des différents organismes. Dans le cas contraire, il faudrait des systèmes clos comme c'est le cas en physique ; or, l'organisme n'est pas un système fermé. Ensuite, l'organisme est à comprendre comme un Tout non comme un ensemble de parties. Canguilhem fournit un exemple précieux : la section de la moelle épinière chez le chat ou le chien a pour conséquence un état de choc caractérisé par l'abolition des réflexes. L'automatisme qui résulte de la période de récupération n'est pas un "rétablissement " mais un autre automatisme. A quoi bon pourrait-on dire, infliger de terribles souffrances à des animaux sachant que d'autres espèces auront d'autres réactions? Enfin le principe d'irréversibilité condamne par avance toute tentative de répétition et de reconstitution d'un phénomène. En d'autres termes, le constat et l'observation d'un phénomène n'attestent en rien de sa reproductibilité ${ }^{11}$.

II s'agit bien pour CL Bernard d'adhérer à une conception déterministe et matérialiste de la vie : du fait que l'être vivant est régi par les lois de la matière, rien n'interdit de se livrer à des expérimentations. On ne peut donc pas évoquer uniquement l'idée d'un progrès de la science mais d'un dogme scientiste au XIXe siècle. Le positivisme de Bernard peut-il se satisfaire d'une telle conception du vivant ? II est vrai que cette forme de scientisme présente des avantages puisqu'elle fait de la physiologie la reine des sciences biologiques.

Pourtant, Auguste Comte, père du positivisme, ne peut totalement adhérer aux vues de Claude Bernard pour des raisons à la fois épistémologiques et morales. Illustrons par un large extrait des Leçons la critique de Comte :

"La vie est bien moins compatible avec l'altération des organes qu'avec celle du système ambiant; je fais, d'ailleurs, ici complètement abstraction de l'évidente considération sociale qui, non seulement à l'égard de l'homme, mais aussi envers les animaux (sur lesquels nous ne serions, sans doute, nous reconnaître des droits absolument illimités), doit faire hautement réprouver cette légèreté déplorable qui laisse contracter à la jeunesse des habitudes de cruauté, aussi radicalement funestes à son développement moral que profondément inutiles, pour ne pas dire davantage, à son développement intellectuel ${ }^{12}$. "

En premier lieu, il faut considérer le vivant comme un Tout ou comme un système organisé. Rien n'est plus contraire à cette conception que la vision mécaniste et fragmentaire du vivant; comme si la section ou l'altération des parties pouvait contribuer à une meilleure compréhension de l'animal ! Ensuite, Comte se rend bien

\footnotetext{
11 «On voit enfin comment l'irréversibilité des phénomènes biologiques s'ajoutant à l'individualité des organismes vient limiter la possibilité de répétition et de reconstitution des conditions déterminantes d'un phénomène, toutes choses égales d'ailleurs, qui reste l'un des procédés caractéristiques de l'expérimentation dans les sciences de la matière. » Op cité, note 5, P 31.

12 Cours de philosophie positive, III, 40ème Leçon.
} 
compte que les vivisecteurs au XIXe siècle bouleversent un ordre social et moral : d'abord, I'homme n'a aucun privilège par rapport à l'animal et donc aucun droit d'en user comme il lui plaît. Ensuite, c'est une mauvaise éducation morale que de former les futurs vétérinaires à tant de cruauté. Cela témoigne en effet d'un manque de considération pour les plus faibles et donc d'une forme de dépravation morale.

Sans respect pour l'animal, les jeunes médecins à Alfort s'exerçaient en toute liberté en dépit de la souffrance des êtres vivisectés. Ne doit-on pas au contraire exiger du corps médical un haut développement moral, une capacité d'empathie ? Car, toute conduite de cruauté à l'égard d'un homme ou d'un animal traduit un vice ou une perversion.

Toutefois, on peut se demander comment la vivisection au XIXe siècle a pu devenir la norme du Progrès et l'incarnation de la vérité expérimentale ? Est-ce alors la recherche de l'utilité et du profit qui du XIXe siècle au XXle siècle caractérise le devenir des sociétés humaines?

\section{L'utilitarisme et la science : la liberté du chercheur doit-elle être absolue ?}

\section{La science est au service de la société}

La science depuis l'Antiquité a pour finalité d'expliquer le monde. En ce sens, elle est à son origine fille de l'étonnement; $c$ 'est en effet progressivement selon Aristote que les hommes parviennent à vaincre leur ignorance en s'interrogeant sur la cause des phénomènes. A juste titre, on pourrait penser que la finalité de la science est purement désintéressée au service de la vérité. Mais il est évident que d'autres enjeux peuvent être dévoilés et mis à jour. Ainsi, c'est au nom d'un dogme religieux que les conceptions de Galilée, Copernic, Bruno furent combattues. L'anthropocentrisme constitue l'idéologie dominante. Mais il est vrai que la science au cours de son histoire n'a cessé de combattre les superstitions et les croyances irrationnelles. Mais la science peut paradoxalement se transformer en religion si elle ne fait pas de la vérité la norme de sa recherche. Et c'est sans aucun doute au XIXème siècle que la science sombre dans l'excès, dans le scientisme.

Jean-Yves Bory, dans une thèse remarquable, Science et patience. La polémique sur la vivisection au XIXe siècle $^{13}{ }^{\text {}}$, évoque l'idée de messianisme scientifique fondée sur l'idée de liberté. En effet, les idéaux de liberté, d'égalité, hérités de la révolution de 1789 s'incarnent dans "le rationalisme expérimental " : c'est ainsi que le scientisme défend l'idée de progrès par la raison et par la science. Il va sans dire que le progrès de la société s'accompagne de l'essor des technologies et de la science, notamment la science médicale. C'est pourquoi, la vivisection constitue "l'instrument de

\footnotetext{
${ }^{13}$ Thèse de Doctorat, EHESS 2010.
} 
rationalisation " par excellence ${ }^{14}$. Sans l'ombre d'un doute, le vivisecteur présente ses travaux à l'Académie des Sciences devant une foule de spectateurs : il s'agit de montrer les prouesses de la science à travers le geste parfait et précis du vivisecteur. La vivisection témoigne de la maîtrise du savant dans sa pratique; elle participe de ce fait à un lien social ou tout au moins à la croyance d'une communauté de chercheurs ! L'idée de progrès s'incarne dans la conquête d'un homme nouveau, rationnel et inventif :

"Le vivisecteur est un homme nouveau par sa puissance intellectuelle et son absolu contrôle de ses émotions et il transmet sa nouveauté à ses auditeurs, il en fait aussi des hommes nouveaux ${ }^{15}$. "

L'homme nouveau est issu de la classe bourgeoise; les vivisecteurs comme Claude Bernard et Magendie, Brown-Séquard réalisent leur rêve de réussite sociale par la vivisection. Car, on grimpe aussi bien l'échelle sociale par le commerce que par des études médicales! L'homme nouveau, c'est aussi celui qui fait fi de ses sentiments au nom de la Raison, du Progrès. Peu importe alors le spectacle d'animaux torturés puisque le Progrès exige de tels sacrifices ! Il est vrai que le modèle dominant de cette société est le désir d'un contrôle absolu sur toutes choses, quitte à écraser et à exploiter les plus faibles. Car la domination ou l'exploitation de l'homme - notamment la figure du prolétaire - ou de l'animal résulte de la recherche d'un avantage en vue de son bien-être ou de sa santé. Or, il n'est pas certain que le spectacle de la cruauté de la vivisection puisse avoir une utilité. Mais il est certain qu'il faut s'entendre sur le terme " utilité. »

A juste titre, Jean-Yves Bory défend l'idée que la vivisection n'est plus « seulement une pratique scientifique mais un acteur du lien social. " En effet, la vivisection en tant que pratique médicale participe au Progrès de la société. II n'est pas anodin qu'un journal de l'époque Le Temps évoque "la science, le progrès, la vérité "! Cette communauté de médecins, de vivisecteurs voit dans cette nouvelle pratique sociale un moyen de promotion car il suffit de rappeler que la vivisection ne sert qu'à justifier sa domination sur les plus vulnérables (comme toute forme d'exploitation animale). Cette idéologie du progrès justifie en conséquence l'exploitation des plus faibles. II est toutefois paradoxal de constater que les idéaux de liberté et d'égalité puissent engendrer autant d'injustice et de misère. II faut donc faire l'hypothèse que les vivisecteurs cautionnent le rêve de la classe bourgeoise ascendante, celle de parvenir à un meilleur statut social quitte à exploiter les plus faibles au nom du Progrès ! Mais il s'agit clairement d'une idéologie " scientiste » : au nom du progrès, du patriotisme, les vivisecteurs remettent en cause les sentiments d'humanité les plus élémentaires : la cruauté de la vivisection devient " un mal nécessaire »! C'est bien au nom de cette

\footnotetext{
${ }^{14}$ Op cité P 186, note 8.

${ }^{15}$ Op cité P 189 , note 8 .
} 
nouvelle science que la cruauté de cette pratique médicale doit être acceptée ; notons toutefois que des protestations contre la vivisection ont toujours existé.

Mais la force de la vivisection ne vient pas uniquement des médecins ni des distinctions honorifiques comme celle obtenue par Claude Bernard en $1851^{16}$; elle résulte également du poids des institutions. II faut en effet insister sur l'idée de choix de société au XIXe siècle : en effet, ce sont les institutions, les grandes écoles, I'Ecole pratique de médecine qui font la gloire de la médecine expérimentale. Le savant au XIXe siècle bénéficie de toute la légitimité des institutions en place. C'est sans doute pourquoi - comme le souligne avec raison Jean-Yves Bory -, le vivisecteur dispose d'une liberté totale au sein de sa pratique; il faudra bien des protestations, notamment des antivivisectionnistes anglais pour que des mesures de protection à l'égard des animaux soient prises.

La force du scientisme au XIXe siècle résulte donc d'une idéologie qui concilie les intérêts de la science à ceux de la politique : la vivisection est en effet le moyen pour les vivisecteurs de faire montre d'une maîtrise absolue sur les êtres vulnérables; mais elle se fait au prix de la souffrance et de la douleur des animaux. II reste paradoxal de se rendre compte que la promotion sociale des médecins repose sur l'exploitation des êtres fragiles, les animaux incapables de se défendre ; sur un plan social, ce sont les prolétaires qui sont victimes de l'industrialisation. Est-ce à dire que l'organisation rationnelle d'une société se fait au prix de l'exploitation des «faibles »?

La science perd de sa pureté et de sa vérité quand elle se transforme en idéologie. Au XIXe siècle, la Raison érigée en idole doit combattre les sentiments au nom du Progrès ${ }^{17}$. C'est pourquoi, toute sensibilité est écartée car elle est signe de faiblesse. La douleur ou la souffrance de l'animal n'est pas prise en considération car la bête n'éprouve aucun sentiment selon une conception mécaniste et cartésienne dans sa version vulgarisée. Mais il faut souligner l'hypocrisie des vivisecteurs : d'abord les expérimentations faîtes sur les animaux se font dans des laboratoires insalubres et secrets $^{18}$, ensuite, les cordes vocales des animaux sont souvent sectionnées afin d'éviter les cris et enfin la découverte des anesthésiques à la fin du XIXe siècle ne permettra, faute d'un usage correct, de mettre fin à la douleur des animaux. Or, devant l'Académie des sciences, certaines expériences sont sélectionnées afin de ne pas heurter la sensibilité du public. II va sans dire que la pratique de la vivisection s'est faite à l'insu des animaux et du public, dans des conditions déplorables.

\footnotetext{
${ }^{16}$ Prix de physiologie expérimentale en 1851.

${ }^{17}$ Tristesse exprimée par George Sand après l'échec de la révolution de 1848.

${ }^{18}$ Les pratiques de CL Bernard furent la plupart du temps dénoncées par les gens du quartier ; c'est pourquoi, le médecin doit s'assurer de l'aide d'un commissaire...
} 
Il reste à savoir si la science devenue idéologie peut être considérée comme légitime lorsqu'elle bafoue des valeurs comme la compassion et l'humanité pour les êtres vulnérables.

2. L'utilité contre la cruauté : le combat des antivivisectionnistes

II faut d'emblée rappeler les propos d'Auguste Comte au début de cet article : la vivisection est dangereuse d'un point de vue moral car elle conduit à une forme de dépravation. En effet, elle bouleverse les valeurs morales et condamne toute forme d'empathie et de compassion à l'égard des êtres vulnérables. L'idée républicaine de progrès doit être combattue sur le terrain politique. Car la lutte pour la justice sociale passe nécessairement par la protection de ceux qui sont exposés à des risques. Or précisément, la vivisection constitue un acte de cruauté pour les êtres faibles.

II n'est donc pas étonnant que les antivivisectionnistes au XIXe siècle comptent dans leur rang des féministes, des progressistes et de grands défenseurs des causes humaines et animales - Victor Hugo et Victor Schoellcher. II va sans dire que le combat des antivivisectionnistes est politique tant par son souci de justice que par sa contestation de l'ordre républicain. Il s'agit en quelque sorte d'une autre interprétation de l'héritage de la Révolution française, héritage qui doit se démarquer de l'ordre bourgeois. Les antivivisectionnistes contestent l'idéal républicain :

"L'antivivisection relève du mouvement progressiste et perpétue le vrai sens de la révolution française contre les dévoiements du républicanisme et de l'autoritarisme personnel ${ }^{19}$. "

II existe un refus chez les féministes anglaises, dans la lutte contre la vivisection, de l'autorité masculine notamment dans le domaine médical. Force est de constater que la profession médicale est masculine ; les vivisecteurs sont des hommes fiers de leur pouvoir. Frances Power Cobbe, Marie Huot, Anna Kingsford, Maria Deraismes sont des femmes libres qui entendent faire de leur combat contre la vivisection une lutte pour la justice et l'égalité. C'est ainsi que Kingsford bouleverse les mœurs de son temps par ses études de médecine et son refus de faire de la vivisection un art !

Ces femmes, loin des conventions sociales admises, se montrent déterminées et inventives dans leurs actions et leurs prises de position : c'est ainsi que Marie Huot n'hésite pas à perturber au Collège de France en 1883 une démonstration sur un petit singe de Brown- Séquard (successeur de Claude Bernard). Il faut bien entendu souligner que la Ligue antivivisectionniste à laquelle adhère Huot entretient des rapports très étroits avec les anarchistes comme Louise Michel et les féministes révolutionnaires, les socialistes. En effet, la lutte contre la vivisection est une lutte contre l'esclavage qu'elle résulte de l'exploitation des prolétaires ou des animaux !

${ }^{19}$ Op cité note 8, P 238. 
C'est pourquoi, la Ligue antivivisectionniste est abolitionniste puisqu'elle s'oppose à l'existence de la vivisection comme toute forme d'esclavage. D'autre part, des liens étroits existent entre la lutte contre la vivisection et la société de théosophie : Anna Kingsford entretient des relations étroites avec Helena Petrovna Blavatsky. La vision philosophique de la théosophie est compatible avec le respect de la nature, de la vie animale. C'est pourquoi, la lutte contre la vivisection participe d'un respect pour les êtres. Mais il faut bien remarquer une opposition fondamentale entre une science utilitariste et une philosophie spiritualiste : c'est ainsi que Anna Kingsford refuse la séparation de l'homme avec d'autres espèces au nom d'un principe philosophique.

En revanche, la Société française contre la vivisection menée par Bishop apparaît plus modérée : elle entend provoquer une réaction des pouvoirs publics sur les dangers de la vivisection. Ensuite, elle souhaiterait une meilleure législation contre la vivisection. II est logique dans cette perspective que la Société française tente de concilier les impératifs de la science avec le principe d'humanité. C'est ainsi que ce mouvement antivivisectionniste entend déployer des arguments scientifiques et constructifs. Les objectifs visés par la Société française sont donc clairs; il faut réduire le nombre d'expériences pratiquées sur les animaux et il faut faire usage des anesthésiques afin de diminuer la souffrance des animaux. En revanche, la Ligue antivivisectionniste est beaucoup plus radicale et $n^{\prime}$ hésite pas à s'infiltrer dans les laboratoires, les conférences afin de jeter le trouble parmi les vivisecteurs.

II existe bel et bien un mouvement de contestation de la vivisection au XIXe siècle, de la SPA (dont le discours montre une certaine hypocrisie) en passant par la Société française contre la vivisection et la Ligue antivivisectionniste. La question des abus relatifs aux animaux fait l'objet de discussion et de controverses entre les partisans de la vivisection et les opposants. C'est bien-sûr la Presse qui joue un rôle crucial puisqu'elle est à même de relayer l'information concernant la cruauté de la vivisection. Il est vrai alors que le discours se cristallise autour de la question des abus et de l'inutilité de la vivisection. Face à ces critiques, les partisans de la vivisection, les médecins se défendent de commettre des abus tout en accusant les autres! D'autres plus fortunés organisent des prix en Angleterre afin de récompenser les meilleurs ouvrages contre la vivisection...

Force est de constater la nécessité de gagner le public à sa cause : les scientifiques ont toujours tendance à minimiser la souffrance animale alors que les spectateurs refusent toute douleur d'un être vivant même au prix de la science. Les vivisecteurs donnent une image caricaturale des défenseurs de la cause animale : les militantes sont souvent considérées comme des hystériques ou bien des êtres ayant une sensibilité exacerbée ! II s'agit toujours d'opposer d'une part la nécessité de la vivisection pour le progrès de la science et d'autre part, la cruauté de cette pratique ! Les vivisecteurs justifient le sacrifice de "quelques " animaux pour le bien-être de tous alors que les antivivisectionnistes mettent en avant l'inutilité de cet "art». Ainsi, face à la justification de la vivisection, un certain Dehais se moque des prétentions de la 
médecine expérimentale à vouloir étudier l'homme moral dans "les organes palpitants d'un chien " (expression de CL Bernard) alors qu'il s'agit uniquement de flatter son amour-propre!

Il faut toutefois souligner l'hypocrisie des vivisecteurs et des vétérinaires; paradoxalement, ils sont la plupart du temps membres de la SPA afin d'être reconnus dans leur activité. Mais ils se montrent prudents quant à la valeur scientifique de la vivisection tout en la pratiquant ${ }^{20}$ ! Ce pouvoir des médecins et des vivisecteurs fut bel et bien contesté par les antivivisectionnistes. Les valeurs utilitaristes de la science au XIXe siècle ne peuvent satisfaire le besoin de justice et de spiritualité des hommes. C'est pourquoi, il est injustifié moralement pour des féministes et des socialistes comme Anna Kingsford et Marie Huot de sacrifier la sensibilité et la compassion pour I'utilité et la science.

Au XXle siècle, dans des sociétés démocratiques, on pourrait supposer qu'il existe un encadrement législatif des expérimentations animales. Existe-t-il un droit européen relatif à la protection animale ? Et surtout, est-il justifié d'évoquer à propos de la vivisection d'une protection et non d'une abolition pure et simple ?

\section{La protection des animaux d'expérimentation}

1. Le droit européen et les mesures relatives à la protection des animaux

Il est vrai d'affirmer que l'expérimentation animale - terme utilisé par les scientifiques et les juristes - est règlementée par des textes européens, notamment la Convention européenne sur la protection des animaux vertébrés; d'autre part, il existe bien un " droit de l'expérimentation animale » en France présent dans le Code rural. Mais il ne peut à notre sens exister de respect pour l'animal tant que ce dernier n'aura pas un statut juridique : or, la définition du Code civil fait de l'animal " un bien " définition distincte de celle du Code pénal qui le caractérise comme un être sensible ayant droit à une protection. A ce titre, la dignité de l'animal n'est pas reconnue ce qui lui interdit d'avoir des droits; tout au plus une protection. Certes, des comités d'éthique ont été créés en France depuis les années 80 ; mais ils constituent des recommandations sans entraver la liberté d'expérimenter des chercheurs. Ainsi, la France a adopté le principe de responsabilité personnelle des chercheurs sans légiférer : on peut évidemment se demander à quoi sert de faire appel à la bonne conscience des chercheurs sans encadrer juridiquement un principe éthique !

Illustrons notre propos par la Directive 2110/63/UE relative à la protection des animaux utilisés à des fins scientifiques. II semble qu'un véritable compromis entre les expérimentateurs, les pouvoirs publics et l'opinion se manifeste. Mais nous voudrions

${ }^{20}$ Op cité note 8 P 214. 
souligner le caractère contradictoire de certains articles. Conformément à l'Union européenne, il est affirmé que « le bien-être animal est une valeur de l'Union qui est consacrée à l'article 13 du Traité sur le fonctionnement de l'Union européenne. " II est évident que le progrès des sciences a permis de mieux connaître l'animal notamment sa capacité à souffrir et à éprouver de la douleur. Il est donc admis dans l'article 6 de cette directive "la capacité des animaux à éprouver et exprimer de la douleur, de la souffrance, de l'angoisse et un dommage durable. " En toute logique, l'évolution des connaissances scientifiques devrait favoriser la protection des animaux ; mais protéger ne signifie pas nécessairement éradiquer toute forme de douleur. Pourtant, il est reconnu dans l'article 12 que " les animaux ont une valeur intrinsèque qui doit être respectée ». Or, comment un animal peut-il être respecté s'il fait l'objet d'une utilisation ? Il est donc un objet pour la science. II n'est pas vrai qu'on puisse reconnaître une valeur "intrinsèque " à un être qu'on exploite. A notre sens, toutes les mesures de protection de cette directive n'ont pour but que d'admettre la nécessité de l'expérimentation animale !

En effet, l'utilisation de primates non humains est déclarée "nécessaire " à "la recherche biomédicale ": il est pourtant admis que ces animaux ont des hautes aptitudes sociales qui en font des êtres sensibles et conscients. Or, c'est à l'Etat d'indiquer la nécessité d'utiliser de tels animaux! II est précisé qu'il faut se montrer prudent quant à la capture et au transport des singes. Mais c'est ignorer les conditions dans lesquelles sont transportés ces animaux : $1 / 3$ des primates non-humains ne survivent pas lors des transports aériens. A quoi bon évoquer la souffrance des animaux quand leur transport dans des cages et quand leur confinement dans des laboratoires ne conviennent pas du tout à leur nature ?

On pourrait à bon droit évoquer, depuis les travaux de Russel et de Burch ${ }^{21}$ dans les années 60 , la Règle des trois $\mathrm{R}$ à savoir "le remplacement ", "la réduction " et "le raffinement " pour les animaux utilisés à des fins expérimentales. Le remplacement consiste dans l'utilisation de méthodes alternatives dès que cela est possible. La réduction implique de diminuer le nombre d'animaux sacrifiés et donc en conséquence de ne pas reproduire des expériences inutiles. Enfin, le raffinement a pour finalité de réduire la souffrance de l'animal par souci de son intégrité. Il est vrai que le Conseil de l'Europe, l'Union européenne (dont la directive 2010/63/UE) ont adopté ces trois règles mentionnées. II s'agit bien évidemment d'une approche éthique qui n'apparaît pas assez contraignante au regard du droit ${ }^{22}$. En effet, cet humanisme éthique - pour

${ }^{21}$ Les principes de la technique expérimentale humaine: http://3rs.ccac.ca/fr/a-propos/trois-r.html

\footnotetext{
${ }^{22}$ Voir l'article de Géraldine Vial et d'Etienne Vergès, La régulation des recherches précliniques : une analyse humaniste de la protection des animaux d'expérimentation par le droit et l'éthique, RSDA, 1/2009.
} 
reprendre l'expression de Géraldine Vial et d'Etienne Vergès - nous semble insuffisant au regard de la liberté des chercheurs. En d'autres termes, le cadre éthique ne peut à lui seul rendre transparent les procédures d'expérimentation. Jean-Pierre Marguénaud ${ }^{23}$ relate un fait significatif à propos des comités d'éthique et des exigences d'impartialité des chercheurs: la Commission européenne en 2001 souhaitait modifier la directive concernant le droit à l'expérimentation animale. Lors de la proposition de révision en 2008, les chercheurs ont marqué leur hostilité quant à leur liberté d'expérimenter sur les primates non-humains. Face à la pression exercée par les scientifiques, le Parlement européen a modifié certaines propositions de la Commission européenne. De ce fait, les chercheurs pouvaient en toute liberté appliquer la règle des trois $\mathrm{R}^{24}$ ! Or, selon l'auteur, il faudrait $s^{\prime}$ interroger sur la composition des comités d'éthique notamment ceux concernant les risques sanitaires et environnementaux.

Est-il bien raisonnable de laisser à l'expérimentateur le choix d'une démarche humaniste envers les animaux ? II nous semble logique de supposer que tout abus des expérimentateurs est possible dès lors qu'une transparence des actes ne fait pas l'objet d'une stricte réglementation. II ne suffit pas de penser que quelques règles éthiques sont nécessaires à la communauté scientifique pour prendre des décisions. C'est pourquoi, il est impératif que le droit limite la liberté des expérimentateurs afin d'éviter toute forme d'abus.

\section{Protection ou exploitation des animaux de laboratoire ?}

Géraldine Vial et Etienne Vergès ${ }^{25}$ défendent l'idée de similitudes entre la Convention européenne des droits de l'homme et la Convention européenne sur la protection des animaux. Or, nous pensons qu'il n'existe pas de commune mesure entre ces deux types de législation. D’abord, la différence fondamentale réside dans le fait que les hommes ont des "droits » alors que les animaux ne peuvent qu'être protégés. Cette absence de droit pour l'animal - comme nous le rappelions au début de cet article constitue une atteinte à son respect, à sa dignité. II suffit d'ailleurs de comparer le contenu de la Convention européenne des droits de l'homme et celle relative à la protection des animaux. Ainsi, il est stipulé dans l'article 2 sur les droits et libertés que "le droit de toute personne à la vie est protégé par une loi »; sauf dans des cas

\footnotetext{
${ }^{23}$ Expérimentation animale, entre droit et liberté, Quae 2011.

${ }^{24}$ «Inéluctablement, le prix de la liberté des expérimentateurs se paye en termes de défaut de transparence du processus de décision, d'impartialité réduite des organes éthiques de régulation et de lobbying énergétique des zélateurs publics et privés de l'expérimentation animale. » Op cité, note 17, P19.

${ }^{25}$ Op cité, note 16.
} 
impératifs. De même, à l'article 3, il est affirmé que "nul ne peut être soumis à la torture ni à des peines ou traitements inhumains ou dégradants. " La Convention européenne des droits de l'homme reconnaît donc le caractère sacré et inaliénable de la vie. Au nom de la dignité humaine, il est injustifié et contraire à tout principe éthique et juridique de s'en prendre à la vie d'autrui et de torturer. Or, I'animal précisément n'est pas reconnu dans sa dignité puisqu'il n'est pas considéré comme une personne ; tout au plus, il est un " être sensible ». Mais cette qualification ne suffit pas à le respecter. C'est pourquoi, les mesures prises à l'égard de l'animal concernent son bien-être, pas sa condition. Peut-on dans ce cas prétendre que la "légitimité ", la " nécessité » et la "proportionnalité » du but soient l'application d'une méthode " humaniste » comme le prétend la Convention européenne de la protection des animaux?

Il est précisé dans la Convention européenne sur la protection des animaux et dans la directive 2010/63/UE que l'utilisation des animaux doit se faire dans un but précis à savoir la protection de "la santé humaine et animale ainsi que l'environnement ". A ce titre, il est toujours possible de recourir à des méthodes alternatives dès que cela est envisageable. En conséquence, il n'est pas toujours nécessaire de faire usage d'animaux si d'autres expérimentations "in vitro" ou bien encore des simulations informatiques sont possibles. Enfin, la recherche sur l'animal relève d'un calcul de son coût : il s'agit donc de mesurer les avantages sur la santé humaine au regard de la souffrance de l'animal. De ce fait, la directive communautaire du 24 novembre 1986 évoque l'idée de " méthode humaine " dès lors que le sacrifice d'animaux relève d'un minimum de souffrance. Mais le sacrifice d'animaux ne peut en aucun cas contenir l'idée de "méthode humaine " puisqu'il n'admet aucun compromis; à moins de supposer que la science ne vise que des fins utilitaristes! II faut à notre sens faire preuve de cohérence : soit on affirme la nécessité de l'expérimentation animale sans recourir à une méthode humaine; soit on refuse l'expérimentation animale au nom de la souffrance des êtres sensibles. En ce sens, il n'existe pas de compromis entre les impératifs de la science et la reconnaissance de la dignité de l'animal.

Il serait en effet absurde, au regard de la Convention européenne des droits de I'homme, de justifier la torture, la souffrance des êtres humains sous prétexte que cela peut sauver des vies humaines. Les droits de l'homme possèdent en effet une valeur absolue. Ils ne reposent sur aucun marchandage car ce serait remettre en cause leur valeur intrinsèque. Il est donc hypocrite de faire appel à une humanité des traitements des animaux de laboratoire sur l'autel de leur sacrifice ! Car, on ne peut évoquer à la fois le bien-être des animaux et les souffrances qui leur sont infligées.

Bref, les intérêts de la science se heurtent à la prise en compte de la douleur animale. Il ne s'agit pas simplement de réduire cette souffrance mais tout simplement de l'abolir. II faut donc aller au-delà d'une régulation éthique des comportements des chercheurs. Mais au XXle siècle, la science sans aucun doute peut répondre à d'autres 
défis à proportion de ses connaissances : ne peut-on pas espérer d'autres méthodes substitutives quant à l'expérimentation animale?

\section{Vers une abolition de la vivisection}

\section{L'animal n'est pas le modèle biologique de l'homme}

Il serait hasardeux de prétendre à une parfaite similarité entre les réactions physiologiques de l'homme et de l'animal. L'erreur de Bernard et des vivisecteurs au XIXe siècle est d'isoler une partie de l'organisme afin de parvenir à une connaissance secrète d'une fonction biologique. Or, c'est oublier que l'organisme est un Tout et qu'il ne peut par conséquent être analysé par l'ensemble de ses parties. L'expérimentateur est sans doute conscient des limites de ses investigations: il souhaiterait en effet analyser des corps humains mais il sait que cela est contraire à la morale ! Cela n'empêche pas le vivisecteur d'expérimenter sur des guillotinés! Toutefois, la science par l'essor de ses connaissances, ne peut aujourd'hui faire de l'animal le modèle biologique de l'homme. Un modèle peut être considéré comme une représentation schématique de la réalité que l'on veut étudier. A ce titre, on pourrait faire du vol de l'oiseau le modèle de l'avion sans pour autant que les propriétés de l'animal soient comparables à celles de l'avion. Or, l'expérimentation animale se fonde sur l'idée d'une similarité des réactions physiologiques, psychologiques entre l'homme et l'animal. Mais par définition, une espèce se définit par sa propre spécificité.

L'association scientifique "Antidote-Europe » entend dénoncer les excès de la vivisection par une meilleure compréhension des phénomènes biologiques. En premier lieu, il faut bien reconnaître qu'une espèce ne peut être définie exclusivement par ses fonctions biologiques mais qu'elle se caractérise par ses habitudes, son rythme d'activité et de repos; bref une espèce est définie par une niche écologique particulière. En second lieu, les spécificités de chaque espèce - la réaction d'un rat, d'un singe - sont singulières: c'est pourquoi, il n'y a aucune certitude quant à l'identité de réaction face à un stress, face à l'absorption d'une substance chimique. Or, l'erreur de $C L$ Bernard et des vivisecteurs de son temps était de généraliser la fonction biologique d'un organe à celui d'une autre espèce. Le scandale de la médecine s'accompagne donc de la croyance selon laquelle les tests effectués sur les animaux protègent l'homme de toute maladie, de tout effet secondaire. Or, les connaissances acquises aujourd'hui permettent de démentir cette croyance. En effet, selon une étude révélée par le journal Le $\mathrm{Monde}^{26}$, vingt mille patients succombent en France des effets secondaires des médicaments. Evidemment, les scientifiques d'Antidote Europe, preuve à l'appui, montrent bien que les effets secondaires des médicaments n'étaient pas connus! On sait bien, suite à des observations, que le

\footnotetext{
26" 13 novembre 1997.
} 
cancer ne se développe pas de la même manière chez l'homme que chez l'animal. Or, les instituts de recherche encouragent ce type d'expérimentation sur les animaux pour des résultats quasi nuls! Car, les fonctions biologiques sont avant tout déterminées par des protéines, lesquelles vont conduire à la digestion, à la transformation des aliments. On peut donc en déduire que les espèces différentes auront des protéines différentes. Si les réactions physiologiques sont différentes d'une espèce à l'autre, il n'est pas possible d'espérer un modèle prédictif pour l'homme. A titre d'exemple, il suffit de rappeler que certaines substances sont toxiques pour les humains alors qu'elles sont inoffensives pour certains animaux; ensuite le virus comme le sida ne se développe pas chez le chimpanzé alors qu'il est efficient chez l'homme ; enfin, certains produits sont plus cancérigènes pour certaines espèces que pour d'autres. II est en un sens absurde de déclencher des cancers chez des souris sachant que le développement d'une telle maladie n'obéit pas aux mêmes mécanismes que chez l'homme. A juste titre, les scientifiques d'Antidote Europe préconisent de faire des recherches dans le domaine de la biologie moléculaire, lors des dérèglements cellulaires à l'origine des cancers.

Il est donc évident que l'expérimentation animale reste inutile et contestable sur bien des aspects. Elle semble satisfaire les chercheurs soucieux de gloire et de renommée comme au XIXe siècle. Pourtant, des méthodes substitutives existent. Ainsi Antidote Europe a mis en place un programme de toxicologie scientifique (PTS) fondé sur la culture de cellules humaines et la technologie des puces à ADN. Il est possible à partir de ces tests effectués de comprendre par quels mécanismes sont affectées nos cellules par des substances toxiques. Dans le domaine de la biologie biomédicale, des tests sur les tissus humains et les cellules humaines font l'objet d'expérimentation. A cela, on peut ajouter la modélisation informatique : les différentes parties du corps sont ainsi représentées ce qui permet de mieux saisir la complexité du système vivant. Les modèles informatiques seront certainement en mesure de prédire le développement de certains cancers.

II semble raisonnable de mettre fin à l'expérimentation animale en Europe au XXle siècle. Face à la sensibilité de l'opinion publique, des scientifiques, des citoyens ont mis en place en juin 2012 une initiative citoyenne européenne ; "Stopvivisection 27 ". Ainsi, chaque citoyen européen est appelé à voter contre la vivisection : chaque voix porte en effet l'espoir de mettre fin à cette barbarie d'un autre temps !

La question est bien évidemment de savoir si la science et la société ont la volonté politique de mettre fin à la souffrance animale.

\section{Des droits pour les animaux ?}

\footnotetext{
27" http://www.stopvivisection.eu/fr
} 
Il est vrai que les animaux ne sont que des choses ou des biens au regard du droit civil. En conséquence, ce droit de propriété donne un droit absolu de disposer des choses tant économiquement que scientifiquement. Toutefois, I'union européenne reconnaît à l'animal un statut spécifique ; son bien-être constitue une valeur. II semble donc évident de reconnaître la sensibilité de l'animal. Toutefois, la question est bien évidemment se savoir s'il est justifié moralement d'accorder des droits aux animaux. Or, cette question divise car elle conduirait à faire de l'animal l'égal de l'homme. Cette position philosophique antispéciste est notamment défendue par le philosophe australien Peter Singer ${ }^{28}$. Si du point de vue du droit, le statut des animaux est problématique, il convient de se demander si la garantie de leur protection est suffisante. Les débats en philosophie du droit opposent d'une part ceux qui défendent le bien-être des animaux (" welfarisme ») de ceux qui sont abolitionnistes c'est-à-dire favorables à la fin de l'exploitation animale sans que ces positions soient strictes. Certes, on peut améliorer la condition et le bien-être des animaux sans pour autant en faire des sujets de droit. Car, si les animaux sont qualifiés de choses, ils ne pourront n'être que des objets d'exploitation; même si certains actes de cruauté à l'égard des animaux sont sanctionnés.

La position cohérente serait sans aucun doute de faire des animaux des personnes, des sujets de droit. Les enfants par exemple ont des droits même s'ils ne sont pas déclarés responsables de leurs actes. En quelque sorte, on pourrait faire des animaux des " patients moraux. " Le patient moral est bénéficiaire d'un devoir, celui d'être respecté. Mais on peut accepter l'idée de droit sans devoir chez les enfants, les personnes séniles, les animaux. Cette position philosophique très radicale est défendue par le philosophe américain Tom Regan auteur du fameux « les droits des animaux ${ }^{29}$. Les animaux ont en effet une valeur « intrinsèque " qui fait d'eux des êtres à respecter. En effet, l'animal est " sujet d'une vie » : c'est un être sensible qui fait des expériences, qui éprouve du plaisir, de la douleur. Cette expression de la subjectivité de l'animal interdit d'en faire précisément un objet d'exploitation. On comprend d'emblée pourquoi la position de Regan est " abolitionniste » : l'animal n'a pas à être considéré comme un esclave !

\footnotetext{
${ }^{28 "}$ Op cit, note 1.

${ }^{29 "}$ Les droits des animaux, trad E.Utria, Hermann 2013.
} 
Les philosophes utilitaristes comme Bentham ${ }^{30}$ au XIXe siècle à Singer au XXle siècle ont montré que le progrès de la condition de l'animal relevait de la même logique que la libération des femmes, des noirs. Cette philosophie utilitariste s'entend à promouvoir le bien-être animal. Il est certes essentiel de modifier les conditions de vie des poules en cage, des veaux en batterie ; le droit peut y contribuer. Toutefois, nous ne pensons pas que les animaux puissent être considérés comme des sujets de droit si l'on se borne uniquement à améliorer leur condition de vie; c'est une clause nécessaire mais non suffisante. Dans le cas de la vivisection, il semble injustifié moralement de sacrifier des animaux pour le bien-être de l'humanité ! D'ailleurs, cette logique utilitariste ne fait qu'augmenter la souffrance des animaux. L'abolition de la vivisection en Europe s'impose par son évidence! D'autres choix de société sont en effet possibles dans le domaine de la recherche biomédicale. Bien-sûr, il n'y a pas de progrès dans le domaine scientifique sans une évolution du droit, des mœurs. L'animal, reste malgré toutes les avancées juridiques, un être sans statut déterminé.

\section{Conclusion}

La vivisection reste un sujet polémique et d'actualité. Au cours de cette étude, nous avons montré que la vivisection ne pouvait être considérée comme une pratique médicale anodine. En effet, il est nécessaire d'en saisir les enjeux idéologiques: l'expérimentation animale témoigne de la volonté humaine de maîtriser le corps humain à des fins de connaissance. Certes, le but de toute recherche médicale est la santé. Mais il est à notre sens injustifié moralement de sacrifier des êtres sensibles à des fins douteuses. Car, il serait absurde de penser que l'animal est le modèle biologique de l'homme. C'est pourquoi, il est nécessaire de mettre fin à toute idéologie du progrès, de la performance. Toute société démocratique doit tenter d'œuvrer pour la justice ; or, les animaux sont des êtres vulnérables à respecter et non pas seulement à exploiter. L'abolition de la vivisection reste l'un des défis du XXle siècle !

\footnotetext{
${ }^{30}$ « Le jour viendra peut-être où le reste de la création animale acquerra ces droits qui n'auraient jamais pu être refusés à ses membres autrement que par la main de la tyrannie. Les français ont déjà découvert que la noirceur de la peau n'est en rien une raison pour qu'un être humain soit abandonné sans recours au caprice d'un bourreau. On reconnaîtra peut-être un jour que le nombre de pattes, la pilosité de la peau, ou la façon dont se termine le sacrum sont des raisons également insuffisantes pour abandonner un être sensible à ce même sort. Et quel autre critère devrait marquer la ligne infranchissable ? Est-ce la faculté de raisonner, ou peut-être celle de discourir ? Mais un cheval ou un chien adulte sont des animaux incomparablement plus rationnels, et aussi plus causants, qu'un enfant d'un jour, ou d'une semaine, ou même d'un mois. Mais s'ils ne l'étaient pas, qu'est-ce que cela changerait ? La question n'est pas: peuvent-ils raisonner ? Ni : peuvent-ils parler ? Mais: peuvent-ils souffrir ? ».Introduction aux principes de la morale et de la législation, chapitre 17, Vrin, 2011.
} 